\title{
Improving accuracy of derived 12-lead electrocardiography by waveform segmentation
}

\author{
Indra Hardian Mulyadi ${ }^{1}$, Nelmiawati ${ }^{2}$, Eko Supriyanto ${ }^{3}$ \\ ${ }^{1,3}$ School of Biomedical Engineering and Health Sciences, Universiti Teknologi Malaysia, Malaysia \\ ${ }^{2}$ Department of Electrical Engineering, Politeknik Negeri Batam, Indonesia
}

\begin{tabular}{l} 
Article Info \\
\hline Article historys: \\
Received Dec 31, 2018 \\
Revised Jan 27, 2019 \\
Accepted Feb 20, 2019 \\
\hline
\end{tabular}

\section{Keywords:}

Artificial neural network

Derived 12-lead

Electrocardiography

EASI

Personalized ECG

Regression

\begin{abstract}
A number of methods have been proposed to reduce number of leads for electrocardiography (ECG) measurement without decreasing the signal quality. Some limited sets of leads that are nearly orthogonal, such as EASI, have been used to reconstruct the standard 12-lead ECG by various transformation techniques including linear, nonlinear, generic, and patientspecific. Those existing techniques, however, employed a full-cycle ECG waveform to calculate the transformation coefficients. Instead of calculating the transformation coefficients using a full-cycle waveform, we propose a new approach that segments the waveform into three segments: PR, QRS complex, and ST, hence the transformation coefficients were segment-specific. For testing, our new segment-specific approach was applied to six existing methods: Dower's method with generic coefficients, Dower's method with individual (patient-specific) coefficients, Linear Regression (LR), 2nd degree Polynomial Regression (PR), 3rd degree PR, and Artificial Neural Network (ANN). The results showed that the new approach outperformed the conventional full-cycle approach. It was able to significantly reduce the derivation error up to $74.50 \%$ as well as improve the correlation coefficient up to $0.66 \%$.
\end{abstract}

Copyright (C) 2019 Institute of Advanced Engineering and Science. All rights reserved.

\section{Corresponding Author:}

Indra Hardian Mulyadi, School of Biomedical Engineering and Health Sciences, Universiti Teknologi Malaysia, Johor, Malaysia.

Email: indra.mulyadi@fkegraduate.utm.my

\section{INTRODUCTION}

Facts mentioned that around 2.6 million people above 15 years old in Indonesia suffered from coronary heart disease [1]. Around 17.3 million World population in 2013 died caused by cardiovascular diseases [2], including coronary heart disease, heart failure, hypertension, and stroke. Advanced technologies have been implemented to reduce this number and - at the same time - to increase life expectancy. The focuses is prevention [3], including prediction and early diagnosis [4], for instance personalized cardiovascular disease monitoring devices [5].

For diagnosis, cardiologists analyze morphology of the ECG waveform. Each segment in the waveform contains information of specific heart activities [6]. Optimal electrodes location for each segment is different, as investigated by Finlat et. al. [7].They introduced Eigenleads which is useful for pre-diagnosing heart pathologies and for wearable ECG, which requires high signal to noise ratio (SNR). However, for wearable ECG, practical aspects must be considered [8].

The standard 12-lead ECG with ten electrodes has been established as diagnostic reference in hospitals. However, it is impractical for 24-hours monitoring, wearable, and ambulatory applications due to difficulty to attach electrodes and sensitivity to wiring noise and motion artifacts [9]. On the other hand, nonclinical users are not trained to find the proper electrodes location, whereas misplacement of the electrodes 
may lead to misdiagnosis [10]. For these reasons, a number of studies have been conducted to employ a limited set of electrodes and transform the acquired signal to produce 12-lead ECG, which is called derived 12-lead ECG. The leads should be nearly orthogonal, for instance lead I, II, and V2 [11].

A promising technique-called EASI-was demonstrated by Dower et al. [12] and has been implemented in hospital for years. One of its benefits is easiness to find body landmarks to place electrodes Figure 1. Three approaches have been investigated to calculate EASI coefficients: generic, patient-specific, and populationspecific [13]. To obtain maximum accuracy, the coefficients should be patient-specific (personalized coefficients) [14]. To improve EASI coefficients, several techniques have been presented such as Dower's method, Linear Regression (LR), Polynomial Regression (PR), Support Vector Regression (SVR), and Artificial Neural Network (ANN) [9]. In those techniques, the coefficients were calculated by utiizing full cycle (FC) of ECG signal, i.e. all segments.

In this study, we propose a new approach by utilizing different EASI coefficients for each ECG segment; therefore, it is segment-specific (SS). We segmented the waveform into three segments: 1) PRInterval, i.e. P wave and PR segment, 2) QRS complex, and 3) ST interval, i.e. ST segment and T wave. It was hypothesized that the result might minimize the transformation error. The proposed approach, i.e. SS, was then compared with the conventional one, i.e. FC.

\section{RESEARCH METHOD}

In this study, we used EASI lead system to reconstruct 12-lead ECG. Instead of calculating for full cycle ECG, we proposed to segment the ECG waveform into three segments: PR, QRS, and ST as described in Figure 2. So, we have different transformation coefficients for each segment. At the end, the reconstructed lead of segment PR, QRS, and ST were then combined to be a full ECG signal. For instance, $\boldsymbol{I}_{\text {Derived,PR }}$, $\boldsymbol{I}_{\text {Derived,QRS }}$, and $\boldsymbol{I}_{\text {Derived,STT }}$ were combined into $\boldsymbol{I}_{\text {Derived,SS }}$ as formulated in (1). $\boldsymbol{V}_{\text {Derived,SS }}$ represents voltage of lead I to V6.

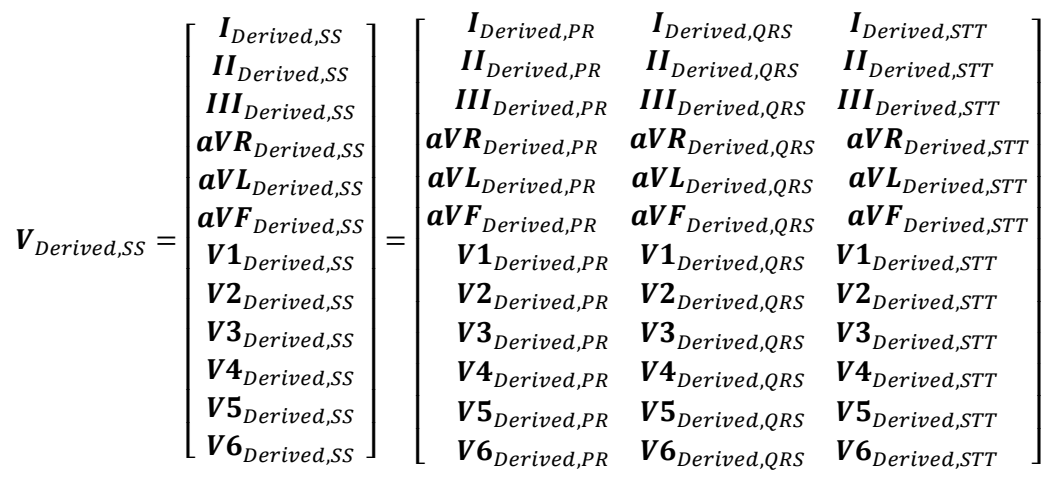

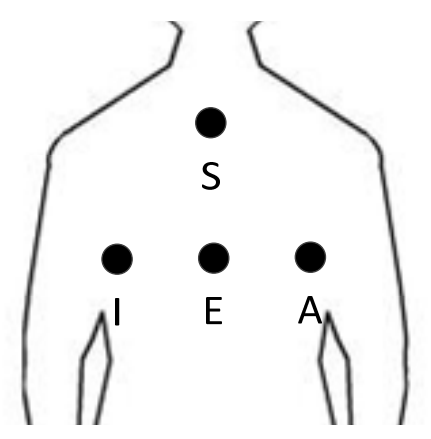

Figure 1. EASI lead system: Electrodes placement

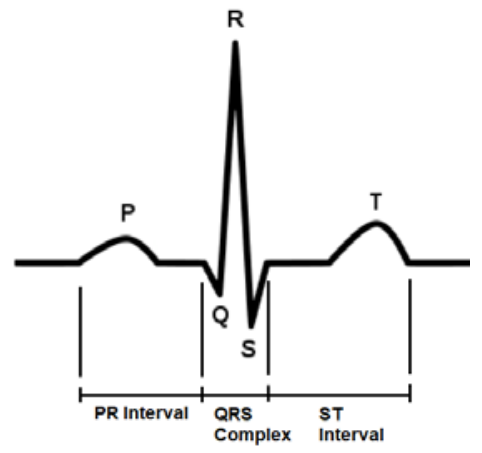

Figure 2. Segmenting one cycle of ECG signal into three segments: PR interval, QRS complex, and ST interval. 
Data sets used in this study were taken from Physionet [15]. Calculation was performed using MATLAB (MathWorks, Natick). To evaluate our new segment-specific (SS) approach, we applied it into six existing methods and compared the result with conventional full cycle (FC) approach. The six existing methods are Dower's method with improved generic coefficients, Dower's method with individual (patient-specific) coefficients, Linear Regression (LR), $2^{\text {nd }}$-degree Polynomial Regression (PR), $3^{\text {rd }}$-degree PR, and Artificial Neural Network (ANN). To simplify, these methods are called method A, B, C, D, E, and F. Method A to E were also presented in our previous work [16].

a. Method A: Dower's method with improved generic coefficients [17]

This method has been applied in commercial EASI 12-lead ECG machines. Generic EASI coefficients were pre-determined statistically from a data set of 983 adult subjects. The (2) was used to calculate derived ECG lead voltage $\left(\boldsymbol{V}_{\text {Derived,A }}\right)$ from three bipolar leads: $\operatorname{ES}\left(\boldsymbol{V}_{E S}\right)$, AS $\left(\boldsymbol{V}_{A S}\right)$, and AI $\left(\boldsymbol{V}_{A I}\right)$ with coefficients of $\beta_{0, A}, \beta_{1, A}$, and $\beta_{2, A}$. As the coefficients are pre-determined, SS and $\mathrm{FC}$ result in a same result.

$$
\boldsymbol{V}_{\text {Derived }, A}=\beta_{0, A} \boldsymbol{V}_{E S}+\beta_{1, A} \boldsymbol{V}_{A S}+\beta_{2, A} \boldsymbol{V}_{A I}
$$

b. Method B: Dower's method with individual (patient-specific) coefficients

Basically, it is similar to method A, but it utilizes individual (patient-specific) coefficients instead of using pre-determined ones.

c. Method C: Linear Regression (LR)

The (3) is a formula used to calculate derived ECG lead voltage $\left(\boldsymbol{V}_{\text {Derived, }}\right)$ from unipolar EASI lead: E $\left(\boldsymbol{V}_{E}\right), \mathrm{A}\left(\boldsymbol{V}_{A}\right), \mathrm{S}\left(\boldsymbol{V}_{S}\right)$, and I $\left(\boldsymbol{V}_{I}\right)$ with coefficients of $\beta_{0, C}, \beta_{1, C}, \beta_{2, C}, \beta_{3, C}$, and $\beta_{4, C}$.

$$
\boldsymbol{V}_{\text {Derived }, C}=\beta_{0, C}+\beta_{1, C} \boldsymbol{V}_{E}+\beta_{2, C} \boldsymbol{V}_{A}+\beta_{3, C} \boldsymbol{V}_{S}+\beta_{4, C} \boldsymbol{V}_{I}
$$

d. Method D: $2^{\text {nd }}$-degree Polynomial Regression ( $\left.2^{\text {nd }} P R\right)$

The (4) is a formula used to calculate derived ECG lead voltage $\left(\boldsymbol{V}_{\text {Derived }, D}\right)$ from unipolar EASI leads: E $\left(\boldsymbol{V}_{E}\right), \mathrm{A}\left(\boldsymbol{V}_{A}\right), \mathrm{S}\left(\boldsymbol{V}_{S}\right)$, and I $\left(\boldsymbol{V}_{I}\right)$ with coefficients of $\beta_{0, D}, \beta_{1, D}, \ldots, \beta_{14, D}$.

$$
\begin{aligned}
& \boldsymbol{V}_{\text {Derived,D }}=\beta_{0, D}+\beta_{1, D} \boldsymbol{V}_{E}+\beta_{2, D} \boldsymbol{V}_{A}+\beta_{3, D} \boldsymbol{V}_{S}+\beta_{4, D} \boldsymbol{V}_{I}+\beta_{5, D} \boldsymbol{V}_{E} \boldsymbol{V}_{A}+\beta_{6, D} \boldsymbol{V}_{E} \boldsymbol{V}_{S}+ \\
& \beta_{7, D} \boldsymbol{V}_{E} \boldsymbol{V}_{I}+\beta_{8, D} \boldsymbol{V}_{A} \boldsymbol{V}_{S}+\beta_{9, D} \boldsymbol{V}_{A} \boldsymbol{V}_{I}+\beta_{10, D} \boldsymbol{V}_{S} \boldsymbol{V}_{I}+\beta_{11, D} \boldsymbol{V}_{E}^{2}+\beta_{12, D} \boldsymbol{V}_{A}^{2}+\beta_{13, D} \boldsymbol{V}_{S}^{2}+ \\
& \beta_{14, D} \boldsymbol{V}_{I}^{2}
\end{aligned}
$$

e. Method E: $3^{\text {rd }}$-degree Polynomial Regression ( $\left.3^{\text {rd }} \mathrm{PR}\right)$

The (5) is a formula used to calculate derived ECG lead voltage $\left(\boldsymbol{V}_{\text {Derived,E }}\right)$ from unipolar EASI leads: E $\left(\boldsymbol{V}_{E}\right), \mathrm{A}\left(\boldsymbol{V}_{A}\right), \mathrm{S}\left(\boldsymbol{V}_{S}\right)$, and I $\left(\boldsymbol{V}_{I}\right)$ with coefficients of $\beta_{0, E}, \beta_{1, E}, \ldots, \beta_{33, E}$.

$$
\begin{aligned}
& \boldsymbol{V}_{\text {Derived,E }}=\beta_{0, E}+\beta_{1, E} \boldsymbol{V}_{E}+\beta_{2, E} \boldsymbol{V}_{A}+\beta_{3, E} \boldsymbol{V}_{S}+\beta_{4, E} \boldsymbol{V}_{I}+\beta_{5, E} \boldsymbol{V}_{E} \boldsymbol{V}_{A}+\beta_{6, E} \boldsymbol{V}_{E} \boldsymbol{V}_{S}+ \\
& \beta_{7, E} \boldsymbol{V}_{E} \boldsymbol{V}_{I}+\beta_{8, E} \boldsymbol{V}_{A} \boldsymbol{V}_{S}+\beta_{9, E} \boldsymbol{V}_{A} \boldsymbol{V}_{I}+\beta_{10, E} \boldsymbol{V}_{S} \boldsymbol{V}_{I}+\beta_{11, E} \boldsymbol{V}_{E}^{2}+\beta_{12, E} \boldsymbol{V}_{A}^{2}+\beta_{13, E} \boldsymbol{V}_{S}^{2}+ \\
& \beta_{14, E} \boldsymbol{V}_{I}^{2}+\beta_{15, E} \boldsymbol{V}_{E}^{3}+\beta_{16, E} \boldsymbol{V}_{A}^{3}+\beta_{17, E} \boldsymbol{V}_{S}^{3}+\beta_{18, E} \boldsymbol{V}_{I}^{3}+\beta_{19, E} \boldsymbol{V}_{E}^{2} \boldsymbol{V}_{A}+\beta_{20, E} \boldsymbol{V}_{E}^{2} \boldsymbol{V}_{S}+ \\
& \beta_{21, E} \boldsymbol{V}_{E}^{2} \boldsymbol{V}_{I}+\beta_{22, E} \boldsymbol{V}_{A}^{2} \boldsymbol{V}_{E}+\beta_{23, E} \boldsymbol{V}_{A}^{2} \boldsymbol{V}_{S}+\beta_{24, E} \boldsymbol{V}_{A}^{2} \boldsymbol{V}_{I}+\beta_{25, E} \boldsymbol{V}_{S}^{2} \boldsymbol{V}_{E}+\beta_{26, E} \boldsymbol{V}_{S}^{2} \boldsymbol{V}_{A}+ \\
& \beta_{27, E} \boldsymbol{V}_{S}^{2} \boldsymbol{V}_{I}+\beta_{28, E} \boldsymbol{V}_{I}^{2} \boldsymbol{V}_{E}+\beta_{29, E} \boldsymbol{V}_{I}^{2} \boldsymbol{V}_{A}+\beta_{30, E} \boldsymbol{V}_{I}^{2} \boldsymbol{V}_{S}+\beta_{31, E} \boldsymbol{V}_{E} \boldsymbol{V}_{A} \boldsymbol{V}_{S}+ \\
& \beta_{32, E} \boldsymbol{V}_{E} \boldsymbol{V}_{A} \boldsymbol{V}_{I}+\beta_{33, E} \boldsymbol{V}_{E} \boldsymbol{V}_{S} \boldsymbol{V}_{I}
\end{aligned}
$$

f. Method F: Artificial Neural Network (ANN) with Backpropagation algorithm utilizing LevenbergMarquardt optimization. The network consists of 88 nodes: one input layer (4 nodes), two hidden layers (72 nodes) with hyperbolic tangent sigmoid activation function, and one output layer (12 nodes) with linear activation function. The network is described in Figure 3. To speed up the calculation, it was divided into 12 networks, one network for each lead. The trained networks produced $\boldsymbol{V}_{\text {Derived,F }}$.

Transformation coefficients of method B to F were calculated from segment-specific (PR, QRS, ST) as well as full-cycle approaches. The results from both were then compared to evaluate their performance. Root mean squared error (RMSE) (6) and correlation coefficient $(r)(7)$ were used as a metric to measure the performances, where $N$ is number of signal samples, $V_{\text {Measured }, i}$ is voltage of measured (original) ECG of $i$-th sample, and $V_{\text {Derived }, i}$ is derived ECG of $i$-th sample. 


$$
\begin{aligned}
& R M S E=\sqrt{\frac{1}{N} \sum_{i=1}^{N}\left(V_{\text {Measured }, i}-V_{\text {Derived }, i}\right)^{2}} \\
& r=\frac{N\left(\sum_{i=1}^{N} V_{\text {Measured }, i} \times V_{\text {Derived }, i}\right)-\left(\sum_{i=1}^{N} V_{\text {Measured }, i}\right)\left(\sum_{i=1}^{N} V_{\text {Derived }, i}\right)}{\sqrt{\left(N \sum_{i=1}^{N} V_{\text {Measured }, i}{ }^{2}-\left(\sum_{i=1}^{N} V_{\text {Measured }, i}\right)^{2}\right) \times\left(N \sum_{i=1}^{N} V_{\text {Derived }, i}{ }^{2}-\left(\sum_{i=1}^{N} V_{\text {Derived }, i}\right)^{2}\right)}}
\end{aligned}
$$

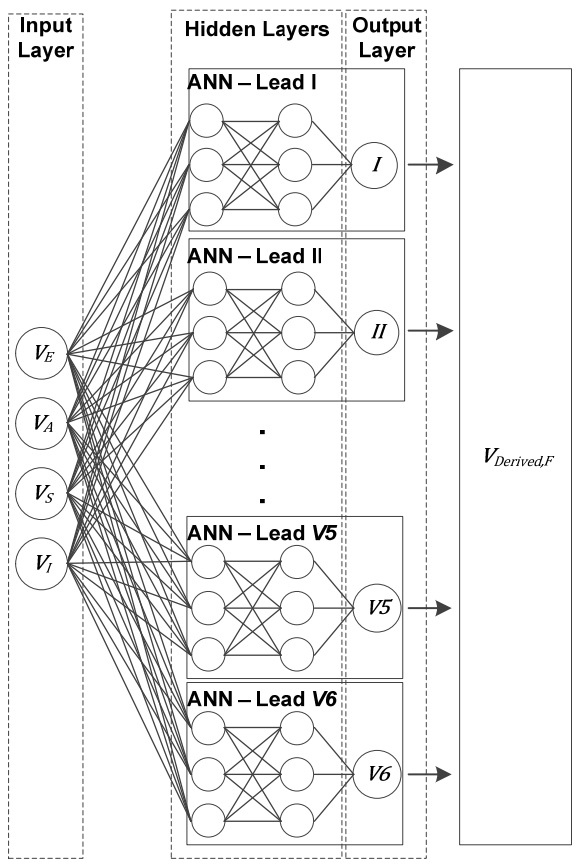

Figure 3. Reconstruction of derived 12-lead ECG from lead E, A, S, and I using 88 nodes ANN (Method F)

\section{RESULTS AND ANALYSIS}

After obtaining segment-specific coefficients for PR, QRS, and ST, we combined coefficients from those three segments to build derived ECG for a whole segment $\left(\boldsymbol{V}_{\text {Derived,SS }}\right)$ using (1). RMSEs comparison of FC and SS is presented in Figure 4. For method B, C, D, and E the new combined segment-specific (SS) outperformed the conventional one (FC). The exception applies to method A, where the coefficients are fixed and pre-determined statistically for the entire segments; hence SS and FC result in a same result [17].

Figure 3 illustrates ECG waveforms from 12 leads (I, II, III, aVR, aVL, aVF, V1, V2, V3, V4, V5, and V6) obtained from measured ECG, derived ECG from conventional FC using method A, and derived ECG from new SS using method E. Table 1 details the comparison of SS and FC in term of RMSE, while Table 2 presents the correlation. From the figure and tables, we conclude that the new segment-specific approach outperformed conventional full-cycle in term of accuracy and correlation, i.e. reducing error significantly up to $74.50 \%$, and improving correlation up to $0.66 \%$. However, the correlation improvement, which indicates similarity degree of ECG morphology, was not significant.

Segmenting ECG waveform into three segments and then calculating the transformation coefficients for each segment results in more accurate and higher correlation derived 12-lead ECG signal. Mathematically, the segmenting can be any, i.e. not necessarily PR, QRS, and ST. However, by segmenting it into the three well-known segments, we might have benefits from the medical perspective, since each segment indicates different information of heart activities [6]. These segments are utilized for different pathological heart diagnosis; for instance, myocardial ischemia can be diagnosed by observing ST segment and T-wave. Segmenting into these three segments is also very useful for pre-diagnosed patients.

Potential drawback of the segment-specific approach would be difficulty of detecting P, Q, R, S, and $\mathrm{T}$ wave automatically; however, several methods to detect these waves have been introduced [18-20]. Besides 
segment-specific, this study also assumed patient-specific for calculation. Although difficult to implement, patient-specific approach is the most accurate than generic or population-specific. The difficulty can be handled by current technologies; for instance, the individual coefficients can be stored in a memory card [11] or Cloud database.

Derivation technique in this paper used data based on Mason-Likar electrode placement. However, for clinical application, a more accurate derivation might be generated from standard 12-lead placement. Mason Likar lead placement may cause misdiagnosis, for instance misdiagnosis and inefficient ablation in predicting outflow tract premature ventricular contraction (OT-PVC) origin [21]

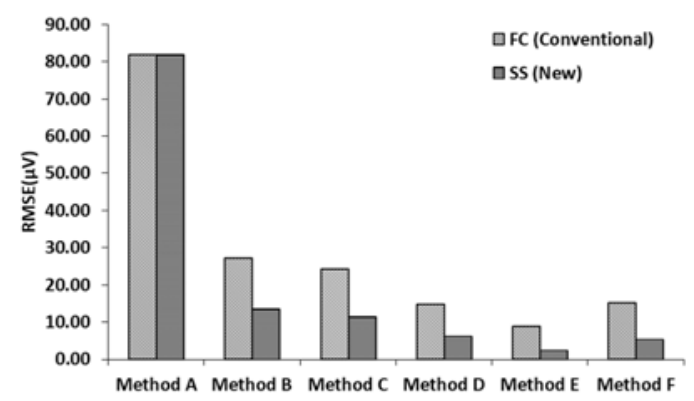

Figure 4. RMSEs of conventional, i.e. FC and our new approach, i.e. SS. SS outperformed FC in term of accuracy

Table 1. Generated RMSEs $(\mu \mathrm{V})$ of 12-lead derived ECG from method A to F. The new segment-specific (SS) outperformed full-cycle (FC) in term of accuracy

\begin{tabular}{cccccccccccc}
\hline \multirow{2}{*}{ Leads } & A & \multicolumn{3}{c}{ B } & \multicolumn{3}{c}{ C } & \multicolumn{1}{c}{ D } & E & \multicolumn{1}{c}{ F } \\
& FC \& SS & FC & SS & FC & SS & FC & SS & FC & SS & FC & SS \\
\hline I & 34.91 & 22.19 & 14.02 & 14.16 & 10.39 & 9.27 & 7.06 & 7.23 & 2.55 & 8.46 & 8.13 \\
II & 113.29 & 38.01 & 15.03 & 28.98 & 9.54 & 18.59 & 3.96 & 10.10 & 1.77 & 26.19 & 7.87 \\
III & 135.79 & 34.73 & 19.39 & 29.14 & 13.95 & 17.76 & 7.56 & 10.58 & 2.52 & 18.95 & 3.99 \\
aVR & 48.99 & 25.83 & 10.83 & 17.55 & 7.13 & 11.70 & 4.30 & 7.01 & 1.79 & 10.00 & 4.86 \\
aVL & 81.27 & 22.09 & 15.16 & 17.74 & 11.34 & 10.69 & 7.04 & 7.53 & 2.37 & 12.10 & 5.10 \\
aVF & 123.86 & 34.67 & 15.87 & 28.19 & 10.76 & 17.58 & 4.89 & 9.69 & 1.76 & 21.45 & 5.53 \\
V1 & 91.98 & 11.75 & 7.50 & 10.04 & 6.42 & 6.86 & 3.10 & 4.55 & 1.17 & 8.25 & 4.01 \\
V2 & 108.53 & 48.97 & 26.26 & 41.97 & 20.91 & 22.35 & 9.91 & 12.90 & 2.98 & 16.49 & 4.83 \\
V3 & 57.22 & 36.54 & 21.34 & 31.93 & 15.98 & 20.53 & 8.07 & 11.26 & 2.83 & 16.23 & 5.51 \\
V4 & 89.23 & 72.10 & 26.38 & 43.35 & 18.13 & 25.86 & 9.79 & 15.98 & 4.66 & 26.64 & 5.04 \\
V5 & 60.46 & 33.96 & 12.33 & 17.71 & 8.23 & 9.02 & 4.80 & 5.97 & 1.80 & 11.45 & 3.62 \\
V6 & 36.69 & 11.15 & 5.14 & 10.37 & 4.25 & 6.51 & 2.29 & 3.56 & 0.91 & 6.43 & 5.13 \\
Average & 81.85 & 32.67 & 15.77 & 24.26 & 11.42 & 14.73 & 6.06 & 8.86 & 2.26 & 15.22 & 5.30 \\
Error Reduction & - & $50.37 \%$ & $52.93 \%$ & $58.83 \%$ & $74.50 \%$ & $65.17 \%$ \\
\hline
\end{tabular}

Table 2. Correlation coefficient of 12-lead derived ECG from method A to F. The new segment-specific (SS)

\begin{tabular}{|c|c|c|c|c|c|c|c|c|c|c|c|}
\hline \multirow[t]{2}{*}{ Leads } & \multirow{2}{*}{$\begin{array}{c}\text { A } \\
\text { FC \& SS }\end{array}$} & \multicolumn{2}{|c|}{ B } & \multicolumn{2}{|c|}{$\mathrm{C}$} & \multicolumn{2}{|c|}{$\mathrm{D}$} & \multicolumn{2}{|c|}{ E } & \multicolumn{2}{|c|}{$\mathrm{F}$} \\
\hline & & $\mathrm{FC}$ & SS & $\mathrm{FC}$ & $\mathrm{SS}$ & $\mathrm{FC}$ & $\mathrm{SS}$ & $\mathrm{FC}$ & $\mathrm{SS}$ & $\mathrm{FC}$ & $\mathrm{SS}$ \\
\hline I & 0.9976 & 0.9970 & 0.9986 & 0.9978 & 0.9988 & 0.9991 & 0.9995 & 0.9994 & 0.9999 & 0.9992 & 0.9993 \\
\hline II & 0.9276 & 0.9938 & 0.9989 & 0.9947 & 0.9994 & 0.9978 & 0.9999 & 0.9994 & 1.0000 & 0.9957 & 0.9996 \\
\hline III & 0.6131 & 0.9589 & 0.9898 & 0.9594 & 0.9909 & 0.9851 & 0.9973 & 0.9947 & 0.9997 & 0.9831 & 0.9993 \\
\hline aVR & 0.9822 & 0.9965 & 0.9992 & 0.9975 & 0.9996 & 0.9989 & 0.9998 & 0.9996 & 1.0000 & 0.9992 & 0.9998 \\
\hline $\mathrm{aVL}$ & 0.9550 & 0.9809 & 0.9923 & 0.9814 & 0.9925 & 0.9933 & 0.9971 & 0.9967 & 0.9997 & 0.9914 & 0.9985 \\
\hline $\mathrm{aVF}$ & 0.7998 & 0.9873 & 0.9976 & 0.9881 & 0.9983 & 0.9954 & 0.9996 & 0.9986 & 1.0000 & 0.9932 & 0.9995 \\
\hline V1 & 0.9949 & 0.9993 & 0.9997 & 0.9993 & 0.9997 & 0.9997 & 0.9999 & 0.9999 & 1.0000 & 0.9996 & 0.9999 \\
\hline $\mathrm{V} 2$ & 0.9643 & 0.9932 & 0.9977 & 0.9935 & 0.9984 & 0.9982 & 0.9996 & 0.9994 & 1.0000 & 0.9990 & 0.9999 \\
\hline V3 & 0.9828 & 0.9946 & 0.9978 & 0.9947 & 0.9987 & 0.9978 & 0.9997 & 0.9993 & 1.0000 & 0.9986 & 0.9998 \\
\hline V4 & 0.9740 & 0.9929 & 0.9985 & 0.9931 & 0.9988 & 0.9975 & 0.9996 & 0.9991 & 0.9999 & 0.9974 & 0.9999 \\
\hline V5 & 0.9910 & 0.9980 & 0.9995 & 0.9980 & 0.9996 & 0.9995 & 0.9999 & 0.9998 & 1.0000 & 0.9992 & 0.9999 \\
\hline V6 & 0.9875 & 0.9988 & 0.9997 & 0.9988 & 0.9998 & 0.9995 & 0.9999 & 0.9999 & 1.0000 & 0.9995 & 0.9997 \\
\hline Average & 0.9308 & 0.9909 & 0.9974 & 0.9914 & 0.9979 & 0.9968 & 0.9993 & 0.9988 & 0.9999 & 0.9962 & 0.9996 \\
\hline Improvement & - & \multicolumn{2}{|c|}{$0.66 \%$} & \multicolumn{2}{|c|}{$0.66 \%$} & \multicolumn{2}{|c|}{$0.25 \%$} & \multicolumn{2}{|c|}{$0.11 \%$} & \multicolumn{2}{|c|}{$0.34 \%$} \\
\hline
\end{tabular}



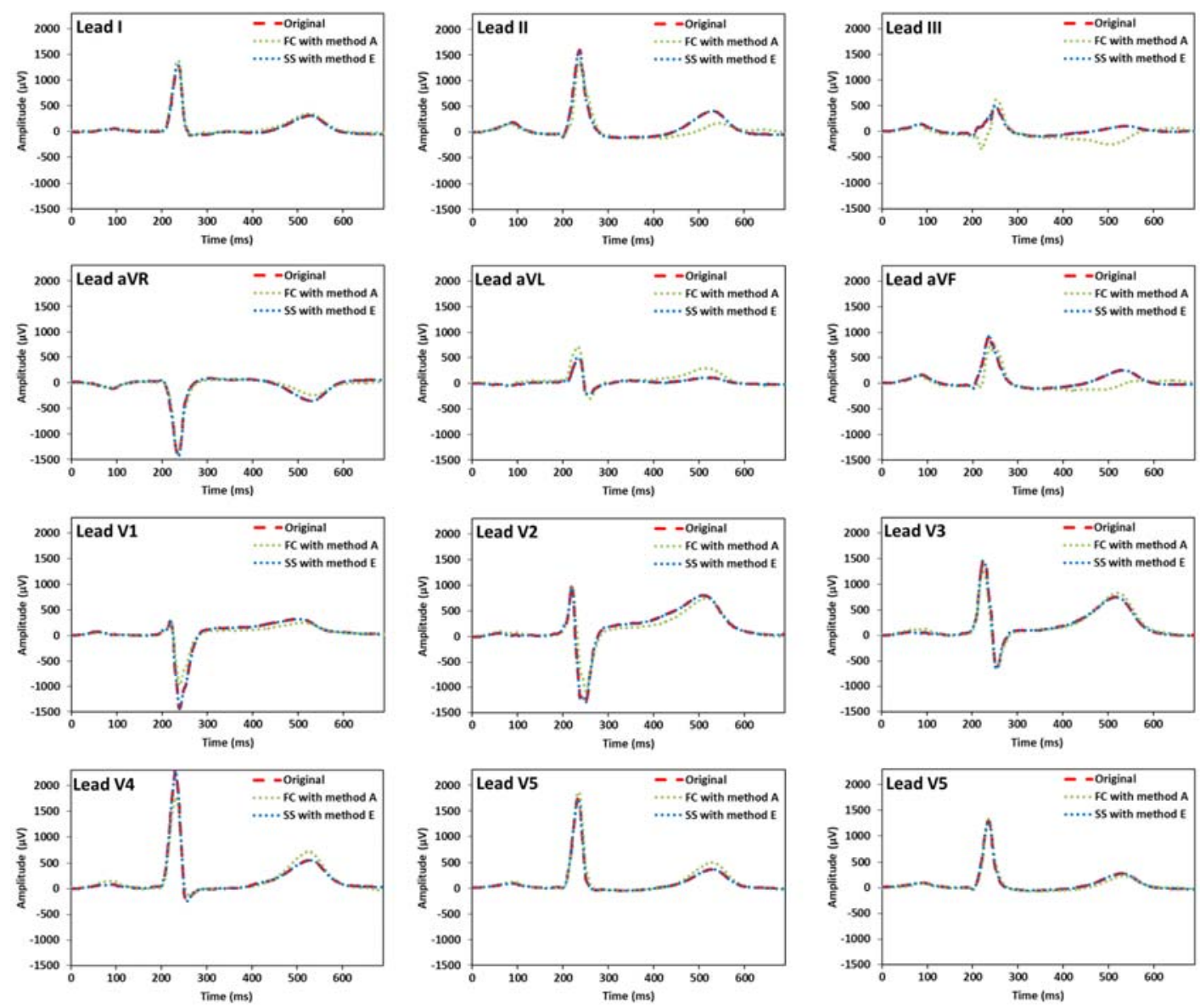

Figure 3. ECG signal: Measured (original), derived from full-cycle (FC) using method A, and derived from segment-specific (SS) using method E. The figure shows that the SS approach results in more accurate derived ECG

\section{CONCLUSION}

Segment-specific approach for deriving 12-lead ECG from a limited set of electrodes has been demonstrated. This approach outperformed the conventional full cycle calculation when compared using several methods (Dower's method with individual (patient-specific) coefficients, LR, $2^{\text {nd }} P R, 3^{\text {rd }} P R$, and ANN). It was able to reduce error significantly up to $74.50 \%$ as well as improve the correlation up to $0.66 \%$. This new approach is promising to provide accurate derived 12-lead ECG for personalized (24-hours monitoring), wearable, and ambulatory ECG. Future works would be implementing this approach for real clinical use.

\section{REFERENCES}

[1] Pusdatin, "Info Data: Situasi Kesehatan Jantung," Kementrerian Kesehatan Republik Indonesia, Jakarta, 2014.

[2] Emelia J. Benjamin et al., "Heart Disease and Stroke Statistics-2017 Update: A Report from the American Heart Association," American Heart Association, 2017.

[3] Victor J. Dzau and Celynne A. Balatbat, "Cardiovascular Research and the National Academy of Medicine: Advancing Progress in Science and Medicine," Circulation Research, vol. 120, pp. 23-26, 2017.

[4] Cuba Gyllensten et al., "Early Indication of Decompensated Heart Failure in Patients on Home-Telemonitoring: A Comparison of Prediction Algorithms Based on Daily Weight and Noninvasive Transthoracic Bio-impedance," JMIR Medical Informatics, vol. 4(1), 2016.

[5] Naresh Vemishetty et al., "Low Power Personalized ECG Based System Design Methodology for Remote Cardiac Health Monitoring," IEEE Access, vol. 4, pp. 8407-8417, December 2016. 
[6] Jakko Malmivuo and Robert Plonsey, "Bioelectromagnetism-Principles and Applications of Bioelectric and Biomagnetic Fields," New York: Oxford University Press, 1995.

[7] Dewar D. Finlay, Chris D. Nugent, Mark P. Donnelly, and Robert L. Lux, "Eigenleads: ECG Leads for Maximizing Information Capture and Improving SNR," IEEE Transactions on Information Technology in Biomedicine, vol. 14(1), pp. 69-78, January 2010.

[8] Indra H. Mulyadi, Jens Haueisen, and Eko Supriyanto, "Practical aspects in surface biopotential electrode placement for smart clothing: A simulation study," in Proceedings of the First International Symposium of Biomedical Engineering (ISBE 2016), 2017.

[9] P. Kaewfoongrungsi and D. Hormdee, "Improving EASI Model via Machine Learning and Regression Techniques," Journal of Telecommunication, Electronic and Computer Engineering, vol. 10, no. 1-5, pp. 115-120, 2018.

[10] Michał Kania et al., "The Effect of Precordial Lead Displacement on ECG Morphology," Med Biol Eng Comput, vol. 52, pp. 109-119, 2014.

[11] Hussein Atoui, Jocelyne Fayn, and Paul Rubel, "A Novel Neural-Network Model for Deriving Standard 12-Lead ECGs From Serial Three-Lead ECGs: Application to Self-Care," IEEE Transactions on Information Technology in Biomedicine, vol. 14(3), pp. 883-890, May 2010.

[12] Dower GE, Yakush A, Nazzal SB, Jutzy RV, and Ruiz CE, "Deriving the 12-lead Electrocardiogram from Four (EASI) Electrodes," Journal of Electrocardiology, vol. 21, pp. S182-187, 1988.

[13] Ivan Tomasic and Roman Trobec, "Electrocardiographic Systems with Reduced Numbers of Leads - Synthesis of the 12-Lead ECG," IEEE Reviews in Biomedical Engineering, vol. 7, pp. 126-142, 2014.

[14] Roman Trobec and Ivan Tomasic, "Synthesis of the 12-Lead Electrocardiogram from Differential Leads," IEEE Transactions on Information Technology in Biomedicine, vol. 15(4), pp. 615-621, July 2011.

[15] Ary L. Goldberger et al., "PhysioBank, PhysioToolkit, and PhysioNet: Components of a New Research Resource for Complex Physiologic Signals," Circulation, vol. 101(23), pp. 215-220, June 2000.

[16] Indra Hardian Mulyadi, Nelmiawati, and Eko Suproyanto, "Segment-Specific EASI Coefficients for Improving Accuracy of Derived 12-Lead Electrocardiography," in 2018 International Conference on Applied Engineering, pp. 1-4, 2018.

[17] Dirk Q. Feild, Charles L. Feldman, and B. Milan Horacek, "Improved EASI Coefficients: Their Derivation, Values, and Performance," Journal of Electrocardiology, vol. 35, pp. 23-33, 2002.

[18] Gustavo Lenisa et al., "P Wave Detection and Delineation in the ECG Based on the Phase Free Stationary Wavelet Transform and Using Intracardiac Atrial Electrograms as Reference," Biomededical Engineering, vol. 61(1), pp. 37-56, 2016.

[19] Chieh-Li Chen and Chun-Te Chuang, "A QRS Detection and R Point Recognition Method for Wearable Single-Lead ECG Devices," Sensors, vol. 17(9), pp. 1-19, August 2017.

[20] Nopadol Uchaipichat, Chusak Thanawattano, and Adisai Buakhamsri, "The Development of ST-Episode Detection in Holter Monitoring for Myocardial Ischemia," Procedia Computer Science, vol. 86, pp. 188-191, 2016.

[21] Suguru Nishiuchi et al., "A Potential Pitfall of the Modified 12 Lead Electrocardiogram (Mason-Likar Modification) in Catheter Ablation of Idiopathic Ventricular Arrhythmias Originating from the Outflow Tract," Europace, vol. 17(12), pp. 1840-1847, December 2015. 